\title{
Nonlinear microscopy for the visualization of calcification and assessment of connective tissue fibers in pseudoxanthoma elasticum
}

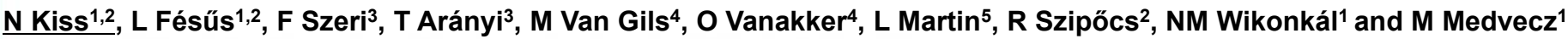

1 Department of Dermatology. Venereology and Dermatooncology. Semmelweis University. Budapest, Hungary

2 Wigner RCP, Institute for Solid State Physics and Optics, Hungarian Academy of Sciences, Budapest, Hungary

3 Research Center for Natural Sciences, Institute of Enzymology, Hungarian Academy of Sciences, Budapest, Hungary

4 Center for Medical Genetics, Ghent University Hospital, Ghent, Belgium

5 PXE Reference Center, Angers University Hospital, Angers, France

\section{Introduction}

Pseudoxanthoma elasticum (PXE, OMIM\#264800) is a rare genetic disorder caused by mutations of the $A B C C 6$ gene in which decreased plasma levels of inorganic pyrophosphate (PPi) lead to ectopic mineralization resulting in severe dermatological, ophthalmologic and cardiovascular complications [1]. Though PXE skin lesions can be identified clinically in most cases, skin biopsy and/or genetic analysis are required to confirm the diagnosis [2]. Components of the dermal connective tissue can be differentiated with nonlinear optical (NLO) techniques, as collagen emits a strong second harmonic generation (SHG) signal given its noncentrosymmetric structure, while elastin as an endogenous chromophore can be visualized by two-photon excitation fluorescence (TPF) [3] [4]

\section{Materials and methods}

Three female and two male PXE patients with a mean age of 53.8 \pm 13.1 years were included in this study. We carried out deep phenotyping, molecular genetic testing and plasma PPi measurement of five patients with PXE [5]. Ex vivo SHG and TPF imaging and conventional histopathological evaluation of their skin was performed and compared to five age- and gender-matched healthy controls. The generated TPF signal is mainly emitted by elastin in the dermis, while SHG displays the collagen structure. Unpaired, two-tailed Student's $t$-test Mann Whitney's U-test and linear regression were used for statistical analysis using GraphPad Prism v6.0 software (*p<0.05), as applicable.

\section{Results}

Phenodex scores can be found in Table 1. Pathological mutations were found in the $A B C C 6$ gene in all patients (Table 2.) Plasma PPi levels of PXE patients were significantly lower compared to controls $(p=0,0005$, Mean $\pm S D$ : $0.232 \pm 0.063$ $\mu \mathrm{M}$ vs $0.947 \pm 0.108 \mu \mathrm{M}$, respectively) (Fig. 1). Papillary dermis and deep layers of the dermis appeared to be unaffected in all patients. However, the mid-dermis of PXE patients exhibited prominent changes (Fig. 2.) We visualized and quantified the degradation and mineralization of elastin fibers and extensive mid-dermal calcium salt deposition as well as the alterations of collagen structure in the mid-dermis of patients with PXE utilizing TPF and SHG, respectively (Fig. 3 and 4 .)
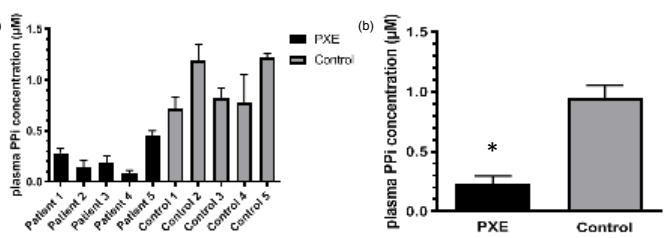

Fig. 1. PPi levels of our PXE patients compared to age- and gendermatched healthy controls. Blood samples were collected and platelet-free plasma was prepared. (a) Plasma PPi levels of all included patients were determined by first converting PPi to ATP, then measuring ATP content in a bioluminescent assay PP concentration of plasma was calculated based on calibration standards and corrected for initial plasma ATP concentration. (b) Plasma PPi levels in PXE vs control patients.

Table 1. Demographic data and Phenodex scores according to Legrand et al. of our patients with PXE.

\section{B}

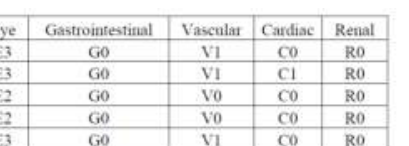

\section{References}

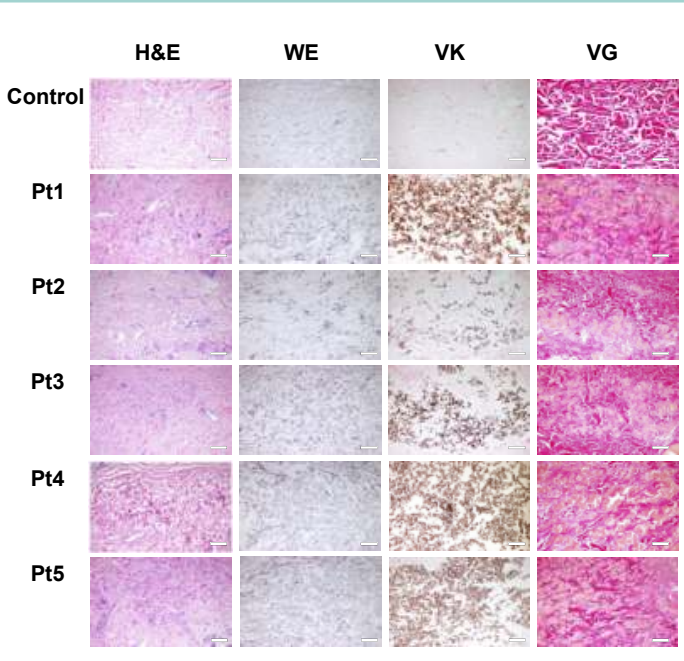

Figure 2. Representative histology images of the mid-dermis of healthy and PXE-affected skin, stained with hematoxylin and eosin (H\&E), Weigert's elastic (WE), von Kossa (VK) and Van Gieson's (VG) stainings. Scale bars display $200 \mu \mathrm{m}$
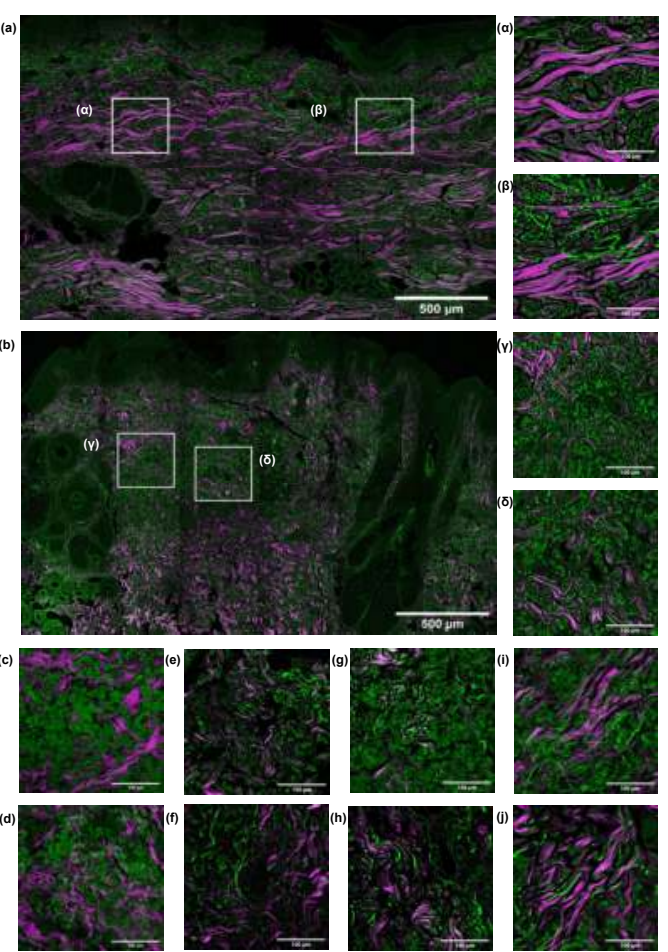

Figure 3. TPF and SHG images of PXE-affected and healthy skin. A $525 / 50 \mathrm{~nm}$ band pass filter was used to separate TPF signal and a $405 / 20 \mathrm{~nm}$ filter for the SHG signal. $420 \times 420 \mu^{2}$ field of views were captured and assembled into $(a, b)$ mosaic images of skin samples with $300 \times 300 \mu m^{2}$ high zoom images of the dermis $(\alpha, \beta, y, \delta)$. (a) Control skin, (b) skin of Patient 3. Representative high zoom inserts are marked with of the middermis (c-j). (c,d) Patient 1, (e,f) Patient 2, (g,h) Patient 4, (i,j) Patient 5 . Scale bars display $500 \mu \mathrm{m}$ for mosaic images and $100 \mu \mathrm{m}$ for high zoom images.

Favre G, Laurain A, Aranyi T, Szeri F, Fulop K, Le Saux O, et al. The ABCC6 Transporter: A New Player in Biomineralization. International journal of molecular sciences 2017;18(9).

Hosen MJ, Lamoen A, De Paepe A, Vanakker OM. Histopathology of pseudoxanthoma elasticum and related disorders: histological hallmarks and diagnostic clues. Scientifica 